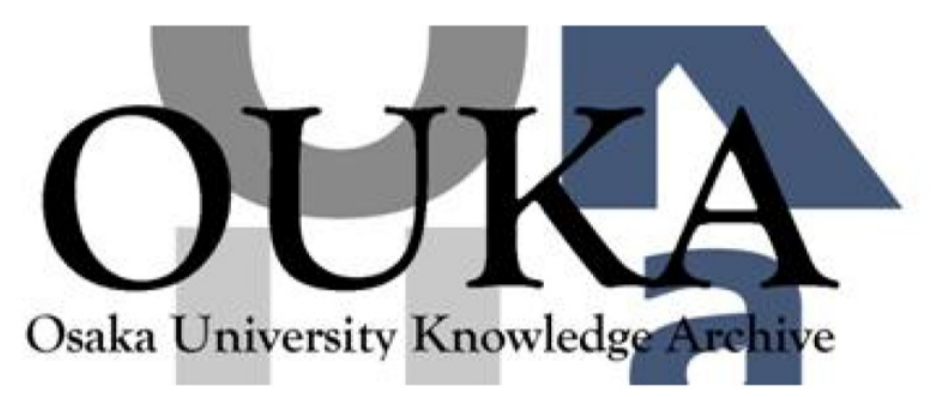

\begin{tabular}{|c|l|}
\hline Title & $\begin{array}{l}\text { A CMOS IF Variable Gain Amplifier with } \\
\text { Exponential Gain Control }\end{array}$ \\
\hline Author(s) & $\begin{array}{l}\text { Cha, Sungwoo; Hirose, Tetsuya; Haruoka, Masaki } \\
\text { et al. }\end{array}$ \\
\hline Citation & $\begin{array}{l}\text { IEICE TRANSACTIONS on Fundamentals of } \\
\text { Electronics, Commun icat i ons and Computer } \\
\text { Sciences. E88-A(2) p. 410-p.415 }\end{array}$ \\
\hline Issue Date & $2005-02$ \\
\hline oaire:version VoR \\
\hline URL & https://hdl. handle. net/11094/51696 \\
\hline rights & copyright $\odot 2005$ IEICE \\
\hline Note & \\
\hline
\end{tabular}

Osaka University Knowledge Archive : OUKA

https://ir. Library. osaka-u. ac. jp/

Osaka University 


\title{
A CMOS IF Variable Gain Amplifier with Exponential Gain Control
}

\author{
Sungwoo CHA ${ }^{\dagger a)}$, Tetsuya HIROSE ${ }^{\dagger}$, Nonmembers, Masaki HARUOKA ${ }^{\dagger \dagger}{ }^{\dagger \dagger}$, Student Member, \\ Toshimasa MATSUOKA ${ }^{\dagger}$, and Kenji TANIGUCHI ${ }^{\dagger}$, Members
}

\begin{abstract}
SUMMARY An intermediate frequency (IF) variable gain amplifier (VGA) with exponential gain control for a radio receiver is fabricated in $0.25-\mu \mathrm{m}$ CMOS technology. The techniques to improve the bandwidth and to reduce temperature dependence of gain are described. The complete VGA is composed of two stages of linearized transconductance VGA and three stages of fixed gain amplifier (FGA). The complete VGA provides a continuous $10 \mathrm{~dB}$ to $76.5 \mathrm{~dB}$ gain control range, an IIP3 of $-11.5 \mathrm{dBm}$ and an $\mathrm{NF}$ of $15 \mathrm{~dB}$ at $40 \mathrm{MHz}$.

key words: variable gain amplifier, VGA, linearized transconductance, linear-in-dB characteristics, exponential gain control, CMOS
\end{abstract}

\section{Introduction}

A variable gain amplifier (VGA) is an indispensable component for intermediate frequency (IF) stage in radio receiver systems. VGA provides a signal whose amplitude is suitable for analog-to-digital converter (ADC) at back-end ana$\log$ circuits in a receiver.

This paper describes a CMOS linear-in-dB VGA for an IF receiver with bandwidth of $40 \mathrm{MHz}$. The VGA using linear-in-dB characteristics is effective for wireless communication systems due to its wide gain range and lowpower operation. There are two types of CMOS linear-indB VGAs. One is a VGA using MOSFETs in square-law as well as exponential-law regions [1], [2]. The other is a VGA using MOSFETs in only square-law region, which approximates an exponential function [3]-[5]. There is difficulty in correctly incorporating the conventional piece-wise modeling of MOSFETs in different two operation regions, required for design of the former [6]. Meanwhile, the techniques described in [3]-[5] apply exponential function of $\exp (x), \exp (2 x)$, or $\exp (4 x)$ to linear-in-dB characteristics, respectively. The VGA using $\exp (2 x)$ [4] is suitable for our target where it has wide gain range and moderate gain variation with control voltage. This type of VGA which linearizes transconductance with DC offset voltage has not been developed for wireless communication. Although it has over $30 \mathrm{~dB}$ wide gain range, much wider range of gain is demanded for the application. Multi-stage connection of

\footnotetext{
Manuscript received June 21, 2004.

Manuscript revised September 17, 2004.

Final manuscript received October 14, 2004.

${ }^{\dagger}$ The authors are with the Graduate School of Engineering, Osaka University, Suita-shi, 565-0871 Japan.

${ }^{\dagger}$ The author is with FURUNO ELECTRIC CO., LTD., Nishinomiya-shi, 662-8580 Japan.

a)E-mail: csw4004@eie.eng.osaka-u.ac.jp
}

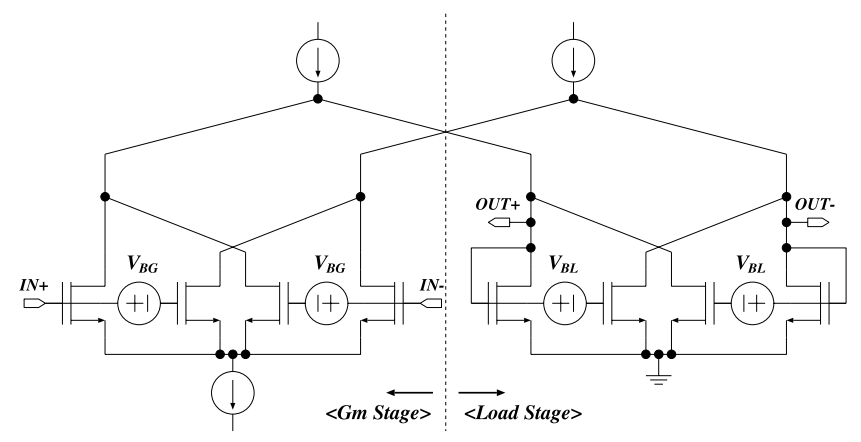

Fig. 1 Basic conceptual schematic of the VGA.

the VGA is one of the solutions to extend gain range but cascade connection degrades frequency characteristics. In this paper, we explain the method to improve VGA bandwidth without gain range trade-off and to reduce temperature dependence of gain. A fixed gain amplifier (FGA) is also designed to enforce overall gain. The complete VGA consists of two-stage modified linearized transconductance VGA, three-stage FGA, and a buffer.

We first describe the principle of the linearized transconductance VGA, followed by the circuit configurations for bandwidth improvement and small temperature dependence of gain.

\section{Circuit Principle and Design}

The linearized transconductance VGA consists of four same size MOSFETs and two DC offset voltage sources in both $G_{m}$ and load stages, as shown in Fig. 1. Gain of the VGA is the ratio of the transconductance of $G_{m}$ stage to that of load stage. Each transconductance varies linearly with DC offset voltage, $V_{B G}$ or $V_{B L}$. Therefore, gain of the VGA, $G_{V G A}$, is expressed by

$$
G_{V G A}=-K \frac{V_{B G}}{V_{B L}},
$$

where $K$ is the size ratio of transistors in $G_{m}$ stage to that in load stage. By making these DC offset voltages vary linearly in opposite direction, the exponential gain control is achieved [4].

Figure 2 shows the designed VGA, FGA and gain control circuit. In the fully-differential VGA circuit, the input signal is amplified by the $G_{m}$ stage, and then is further amplified at the diode-connected load stage. Bias voltages $V_{G D}$ 

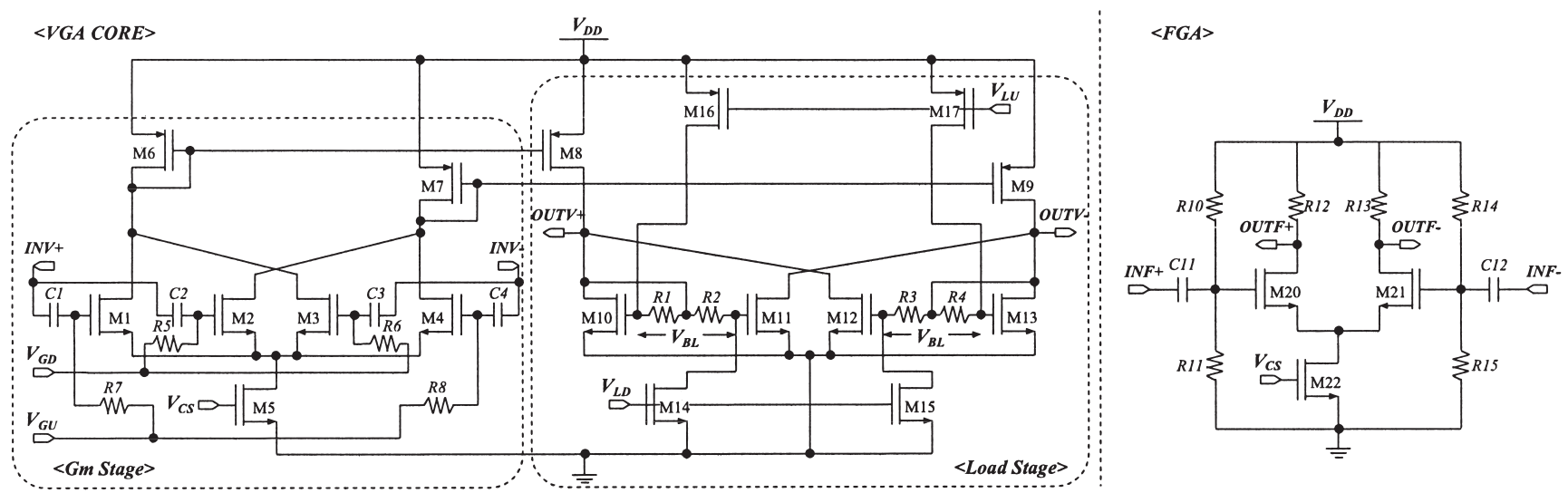

(a)

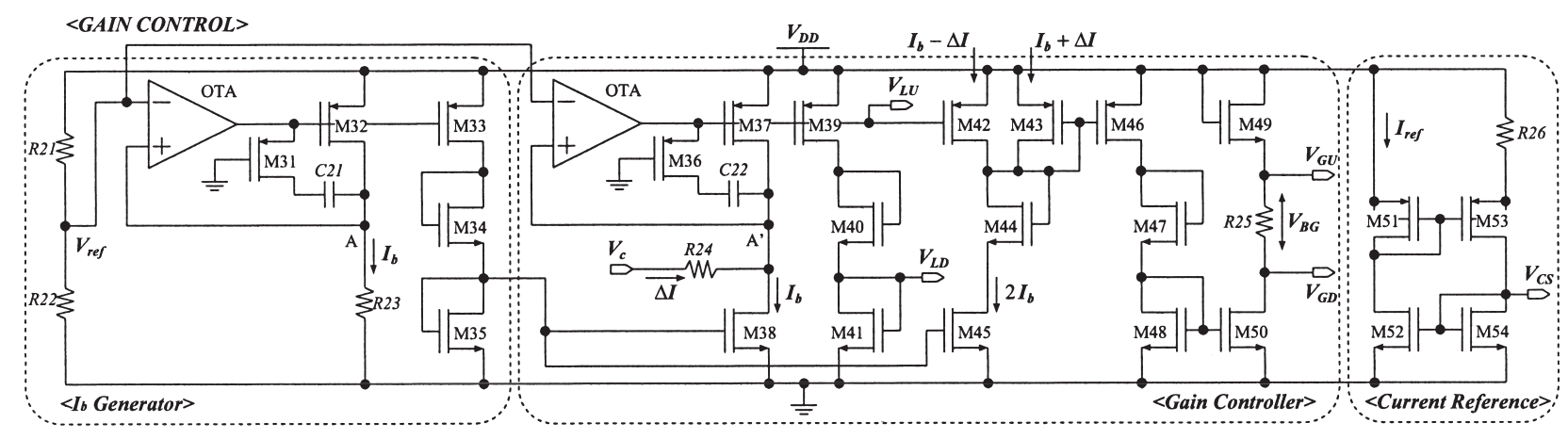

(b)

Fig. 2 Schematics of (a) the VGA and the FGA circuits and (b) the gain control circuit.

and $V_{G U}$ generated at the gain controller provides the DC level of capacitively coupled input of VGA. The offset voltage source, $V_{B G}\left(=V_{G U}-V_{G D}\right)$, is shared in the $G_{m}$ stage by using $R_{5} \sim R_{8}$ of large resistance, while $V_{B L}$ is generated in the load stage.

The FGA is a differential common source amplifier with resistive load to boost absolute gain of the VGA, which is also capacitively coupled to the inputs. The FGA's are placed at the front and back stage of the VGA because the FGA has low noise figure and wide output dynamic range compared with the VGA.

The DC offset voltage source to control VGA gain consists of a resistor and two MOSFETs, and generates the offset voltage by the current through the resistor. The gain controller generates the voltages, $V_{G U}, V_{G D}, V_{L U}$ and $V_{L D}$ by external control voltage $V_{c}$. The offset voltages are given by

$$
\begin{aligned}
V_{B G} & =V_{G U}-V_{G D}=K_{1} R_{25}\left(I_{b}+\Delta I\right), \\
V_{B L} & =K_{2}\left(R_{1}+R_{2}\right)\left(I_{b}-\Delta I\right),
\end{aligned}
$$

where $I_{b}$ is drain current of M38, $\Delta I$ is current through $R_{24}$ and $K_{1}, K_{2}$ are constants given by size ratio of transistors in the gain controller. $I_{b}+\Delta I$ flowing through diode-connected M43 is generated using current sink, M45, of $2 I_{b}$ and current source, M42, of $I_{b}-\Delta I$. From eq.(1), $G_{V G A}$ is determined by
$V_{B G}$ and $V_{B L}$ which are oppositely varied with $\Delta I$. The VGA gain control behavior of $(1+x) /(1-x)$, where $x=\Delta I / I_{b}<1$, emulates exponential function of $\exp (2 x)$.

For the applications of wireless communication, two VGA's have been cascaded to increase gain control range. The cascade connection, however, limits the bandwidth so that a new method to improve the bandwidth has been used as follows. The dominant pole at output node of the VGA is attributed to a resistor and drain capacitance of MOSFETs in the $V_{B L}$ circuit, gate capacitance of diode-connected MOSFET and output capacitance. There is little bandwidth improvement by reducing the resistance due to the inverse relationship between the resistance and drain capacitance under constant $V_{B L}$. Moreover, this leads to the increase of dissipation current in the $V_{B L}$ circuit. In order to increase $3 \mathrm{~dB}$ bandwidth of the VGA, the resistor in the $V_{B L}$ circuit is divided into two equal resistors, $R_{1}$ and $R_{2}\left(R_{3}\right.$ and $R_{4}$ ), and the output node is connected to the center node between two resistors instead of the gate of M10(M13) (diodeconnected load). Similar approach for high speed current mirror, which is a bandwidth improvement technique by introducing a resistor between gate and drain node of diodeconnected MOSFET, has been reported [7], while our approach is that by dividing a already existing resistor in DC 
offset voltage source. Intuitively, by connecting the output node to the center of DC offset voltage source (center-tapped load), the resistance at output node becomes half compared to the case of diode-connected load. Therefore, it is conceivable that the pole and zero frequencies at the output node are doubled by switching just node connection. From the equivalent circuits of the conventional and proposed load stages shown in Fig. 3, their pole frequency, $p$, and zero frequency, $z$, are derived as

$$
p=\frac{X_{2} \pm \sqrt{X_{2}^{2}-4 X_{3}\left(g_{m 1}-g_{m 2}\right)}}{2 X_{3}}, z=\frac{1}{X_{1}},
$$

where

$$
\begin{aligned}
& X_{1}=2 R_{A} C_{A}, \\
& X_{2}=2 C_{A}\left(1+g_{m 1} R_{A}\right)+C_{\text {out }}, \\
& X_{3}=X_{1}\left(C_{A}+C_{\text {out }}\right)
\end{aligned}
$$

in the case of diode-connected load stage, while

$$
\begin{aligned}
& X_{1}=R_{A} C_{A}, \\
& X_{2}=2 C_{A}+C_{\text {out }}, \\
& X_{3}=X_{1} C_{\text {out }}
\end{aligned}
$$

for center-tapped load stage. Here, $C_{A}$ is capacitance at the gate node of M10, $R_{A}$ is resistance of $R_{1}$, and $g_{m 1}$ and $g_{m 2}$ are transconductances of M10 and M11, respectively. Vanished terms from the equation of diode-connected load stage, which are 2 in $X_{1}, g_{m 1} R_{A}$ in $X_{2}$ and $C_{A}$ in $X_{3}$, mean bandwidth improvement with the center-tapped load stage. The ratio of dominant pole frequencies of two load stages is determined by these terms. While there is no bandwidth improvement when $g_{m 1} R_{A} \ll 1$ and $C_{A} \ll C_{\text {out }}$, the ratio

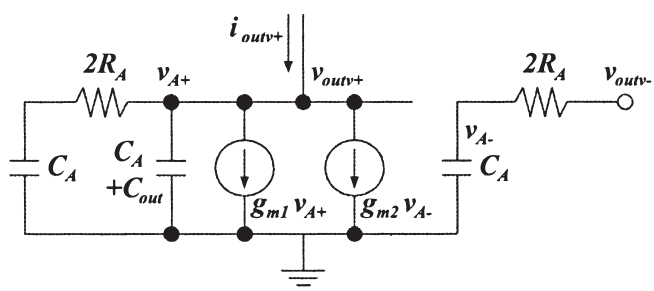

(a)

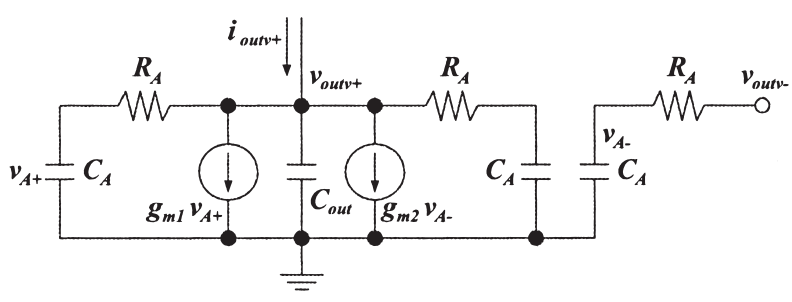

(b)

Fig. 3 Equivalent circuits of (a) conventional diode-connected load stage and (b) proposed center-tapped load stage. can be more than two in the general case of $g_{m 1} R_{A}>1$ and $C_{A} \leq C_{\text {out }}$. It is assumed that all MOSFETs in the load stage have equal drain capacitances and infinite drain resistances for simplicity. Actually, the dominant pole is determined by the circuit elements of $R_{1}$ side from the center tap because PMOS (M16) has larger drain capacitance than NMOS (M14).

The gain of FGA as well as VGA is susceptible to variations in temperature due to temperature dependence of resistors. In order to eliminate the resistance dependence, the $I_{b}$ generator and the beta multiplier current reference [8] have been added to the gain control circuit. VGA gain is a function of $x$ dependent on $R_{24}$ which determines $\Delta I$. The feedback operational transconductance amplifier (OTA) forces voltage at node A(A') to be equal to $V_{\text {ref }}$. Current $I_{b}$ generated by $V_{A}$ across $R_{23}$ in the $I_{b}$ generator is copied to M38. Then, the resistance term in $x$ is cancelled out. The VGA gain and $x$ are independent of temperature.

$$
\begin{gathered}
I_{b}=\frac{V_{r e f}}{R_{23}}, \Delta I=\frac{V_{c}-V_{r e f}}{R_{24}}, \\
x=\frac{\Delta I}{I_{b}}=\frac{R_{23}}{R_{24}} \cdot \frac{V_{c}-V_{r e f}}{V_{r e f}},
\end{gathered}
$$

where $V_{r e f}=V_{D D} \cdot R_{22} /\left(R_{21}+R_{22}\right)$. The VGA gain is dependent on $V_{D D}$ because $V_{\text {ref }}$ is a function of $V_{D D}$. When $R_{21}=R_{22}$ and $R_{23}=R_{24}, G_{V G A} \propto V_{c} /\left(V_{D D}-V_{c}\right)$. This means that the change of $V_{D D}$ affects the VGA gain. Control of $V_{c}$ according to $V_{D D}$ variation by auto gain control (AGC) circuit is one solution for this problem.

On the other hand, the gain of FGA is proportional to the load resistance, $R_{12}\left(R_{13}\right)$. The current $I_{\text {ref }}$ generated by the current reference known as beta multiplier reference is inversely proportional to square of $R_{26}$. Consequently, FGA gain, $G_{F G A}$, is also dependent on resistance ratio but independent of temperature [1], [9] as following equations.

$$
\begin{aligned}
I_{r e f}= & \frac{1}{2 \beta_{51} R_{26}^{2}} \quad\left(W_{53}=4 W_{51}\right), \\
G_{F G A} & =-g_{m 20} \cdot R_{12} \\
& \propto-\sqrt{2 \beta_{20} \frac{I_{r e f}}{2}} \cdot R_{12}=-\frac{R_{12}}{R_{26}} \cdot \sqrt{\frac{\beta_{20}}{2 \beta_{51}} .}
\end{aligned}
$$

The temperature stable complete VGA is composed of a pre-FGA, two-stage modified VGA, two-stage post-FGA and a buffer, and its block diagram is shown in Fig. 4. Source follower was used as a buffer amplifier to load a large output capacitor. As mentioned above, by placing the FGA's

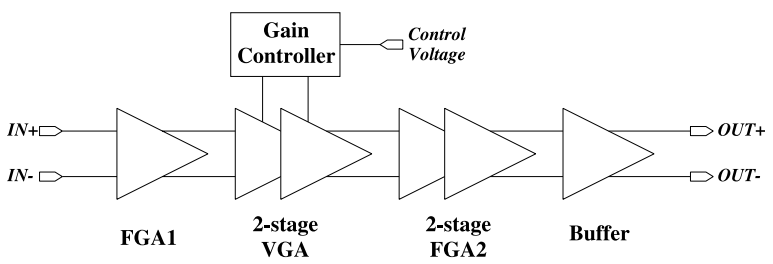

Fig. 4 Block diagram of the complete VGA. 
at both sides of the two-stage VGA, improvements of noise and linearity characteristics have been attempted.

\section{Simulation and Implementation Results}

The circuit has been simulated using HSpice. The VGA with diode-connected load had the $3 \mathrm{~dB}$ frequency of $24 \mathrm{MHz}$ at maximum gain which is the worst case of frequency characteristics. The gain range was varied from $-18 \mathrm{~dB}$ to $11 \mathrm{~dB}$. The bandwidth of the VGA was increased by changing diode-connected load to the center-tapped load, resulting in $83 \mathrm{MHz}$. Therefore, the bandwidth degradation of the two-stage VGA by cascade connection was relaxed. The $3 \mathrm{~dB}$ frequencies of all FGA's and the buffer, wider than $150 \mathrm{MHz}$, affected rarely the complete VGA bandwidth. As a result, the complete VGA had the $3 \mathrm{~dB}$ frequency of $54 \mathrm{MHz}$ and the gain range of $16.5 \mathrm{~dB}$ to $74.5 \mathrm{~dB}$ at $50 \mathrm{MHz}$. The temperature dependence of gain has been also simulated at $-35^{\circ} \mathrm{C}, 27^{\circ} \mathrm{C}$, and $85^{\circ} \mathrm{C}$. Differences from the gain at $27^{\circ} \mathrm{C}$, were obtained within $\pm 3 \mathrm{~dB}$ overall gain range. $\mathrm{A}$ pre-FGA and two stages of post FGAs have been designed as their gains to be about $21.5,10$, and $21 \mathrm{~dB}$, respectively.

The chip has been implemented in $0.25-\mu \mathrm{m}$ CMOS technology for optional $3.3 \mathrm{~V}$ supply voltage with poly and well resistors, and MIM capacitors. For large output swing, the supply voltage was set up to $3.3 \mathrm{~V}$. The chip, shown in Fig. 5, has an active area of less than $0.4 \mathrm{~mm}^{2}$. The circuit has been carefully laid out to ensure matching of devices in all differential circuits and especially matching of four MOSFETs in each $G_{m}$ and load stage of the VGA circuit. The channel length of transistors in the gain control circuit requiring large drain resistance was doubled to that in the other circuits. Poly resistors were used as resistors in the DC offset voltage sources and those related to the temperature compensation due to low parasitic capacitance and fluctuation, while well resistors were used in the DC bias circuits requiring large resistance.

Figure 6 shows the measured frequency response of the complete VGA with control voltage from $0.4 \mathrm{~V}$ to $2.6 \mathrm{~V}$. The complete VGA consumes $21 \mathrm{~mA}$ to $22 \mathrm{~mA}$ under $3.3 \mathrm{~V}$ supply. The upper $3 \mathrm{~dB}$ frequency is $40 \mathrm{MHz}$ at maximum gain, which is slightly smaller than the designed value. This is mainly due to parasitic capacitance of the coupling capacitors, which is added to the load capacitance of each stage. The coupling capacitors in each stage cause the gain reduction for low frequency.

The gain and noise figure (NF) with $V_{c}$ were measured at a signal frequency of $40 \mathrm{MHz}$, as shown in Fig. 7. The gain curve shows continuous linear-in- $\mathrm{dB}$ characteristics of the complete VGA. The gain varies from $10 \mathrm{~dB}$ to $76.5 \mathrm{~dB}$ when $V_{c}$ changes from $0.4 \mathrm{~V}$ to $2.9 \mathrm{~V}$. The average gain range sensitivity is around $26 \mathrm{~dB} / \mathrm{V}$. Gain out of this $V_{c}$ range deviates from extension of the gain curve because some transistors does not operate at saturation region. On the other hand, the NF curve shows different characteristics to the gain curve. At $76.5 \mathrm{~dB}$ gain, $\mathrm{NF}$ of $15 \mathrm{~dB}$ was obtained.

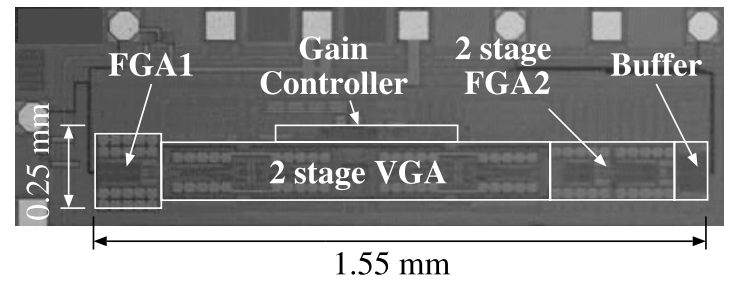

Fig. 5 Photomicrograph of the complete VGA.

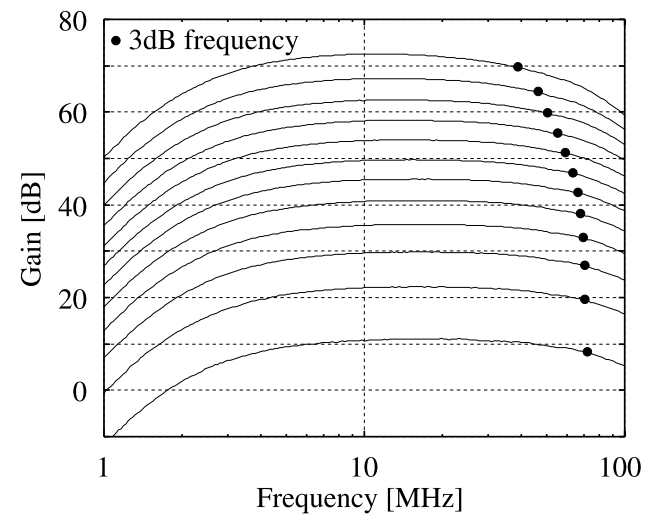

Fig. 6 Frequency responses of the complete VGA increasing control voltage in $0.2 \mathrm{~V}$ step from $0.4 \mathrm{~V}$ to $2.6 \mathrm{~V}$.

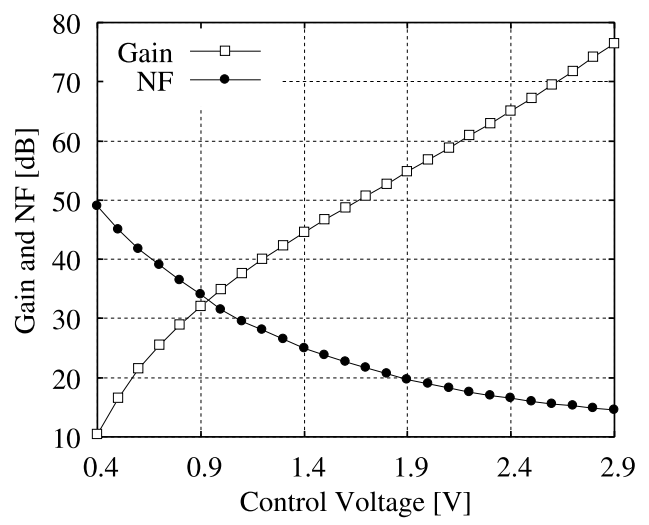

Fig. 7 Gain and NF vs. control voltage at $40 \mathrm{MHz}$ signal frequency.

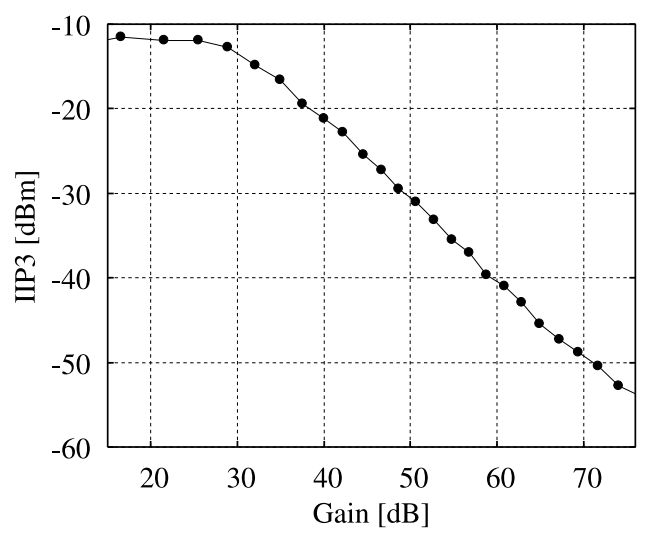

Fig. 8 IIP3 characteristics over the gain control range at $40 \mathrm{MHz}$ signal frequency. 
Table 1 Characteristics of the VGA.

\begin{tabular}{lc}
\hline Bandwidth $(3 \mathrm{~dB})$ & $4-40 \mathrm{MHz}$ \\
Gain range & $10-76.5 \mathrm{~dB}$ \\
$\mathrm{NF}$ & $15 \mathrm{~dB}$ \\
IIP3 @ Gmax & $-53 \mathrm{dBm}$ \\
IIP3 @ Gmin & $-11.5 \mathrm{dBm}$ \\
Supply voltage/current & $3.3 \mathrm{~V} / 21-22 \mathrm{~mA}$ \\
\hline
\end{tabular}

To investigate linearity of the complete VGA, two-tone test at $40 \mathrm{MHz}$ signal frequency with a $1 \mathrm{MHz}$ spacing has been carried out. From the obtained 3rd-order intermodulation distortion (IMD3), the input third intercept point (IIP3) has been calculated [10]. Figure 8 shows the IIP3 characteristics over the gain range. IIP3's of $-11.5 \mathrm{dBm}$ at minimum gain and $-53 \mathrm{dBm}$ at maximum gain were obtained.

Characteristics of the complete VGA are summarized in Table 1.

\section{Conclusions}

An IF linear-in-dB VGA for a radio receiver has been presented. The VGA has been fabricated in $0.25-\mu \mathrm{m}$ CMOS technology. The technique to improve bandwidth of the VGA has been proposed, and the temperature stability of circuit has been also considered. The complete VGA was composed of a pre-FGA, two-stage modified linearized transconductance VGA, two-stage post-FGA and a buffer. The fabricated complete VGA obtained a continuous linearin- $\mathrm{dB}$ gain control range of $10 \mathrm{~dB}$ to $76.5 \mathrm{~dB}$, an IIP3 of $-11.5 \mathrm{dBm}$ and an NF of $15 \mathrm{~dB}$ at $40 \mathrm{MHz}$ signal frequency. The measured results demonstrate that this VGA is suitable for an integrated CMOS radio receiver.

\section{Acknowledgments}

The chip design in this work is supported by VLSI Design and Education Center (VDEC), the University of Tokyo in collaboration with Cadence Design System, Inc. This study is also supported by Japan Science and Technology Agency. Additionally, the authors would like to thank also Synthesis Corp. for their technical support.

\section{References}

[1] T. Yamaji, N. Kanou, and T. Itakura, "A temperature-stable CMOS variable-gain amplifier with $80-\mathrm{dB}$ linearly controlled gain range," IEEE J. Solid-State Circuits, vol.37, pp.553-558, May 2002.

[2] O. Watanabe, M. Ashida, T. Itakura, and S. Otaka, "A 380-MHz CMOS linear-in-dB variable gain amplifier with gain compensation techniques for CDMA systems," IEICE Trans. Electron., vol.E86-C, no.6, pp.1069-1076, June 2003.

[3] P.-C. Huang, L.-Y. Chiou, and C.-K. Wang, "A 3.3-V wideband exponential control variable-gain-amplifier," IEEE International Symposium on Circuits and Systems, vol.1, pp.285-288, 1998.

[4] C.W. Mangelsdorf, "A variable gain CMOS amplifier with exponential gain control," Symposium on VLSI Circuits of Technical Papers, pp.146-149, 2000.

[5] R. Saito, K. Hosoda, A. Hyogo, T. Maruyama, H. Komuraki, H. Sato, and K. Sekine, "A 1.8-V 73-dB dynamic-range CMOS variable gain amplifier," Proc. European Solid-State Circuits Conference, pp.301-304, Sept. 2003.
[6] M. Miura-Mattausch, H. Ueno, H.J. Mattausch, K. Morikawa, S Itoh, A. Kobayashi, and H. Masuda, " $100 \mathrm{~nm}$-MOSFET model for circuit simulation: Challenges and solutions," IEICE Trans. Electron., vol.E86-C, no.6, pp.1009-1021, June 2003.

[7] T. Voo and C. Toumazou, "High-speed current mirror resistive compensation technique," Electron. Lett., vol.31, no.4, pp.248-250, Feb. 1995.

[8] R.J. Baker, H.W. Li, and D.E. Boyce, CMOS Circuit Design, Layout, and Simulation, IEEE Press, 1998.

[9] S. Pavan, Y.P. Tividis, and K. Nagaraj, "Widely programmable high-frequency continuous-time fiters in digital CMOS technology," IEEE J. Solid-State Circuits, vol.35, pp.503-511, April 2000.

[10] B. Razavi, RF microelectronics, Pretice-Hall, 1998.

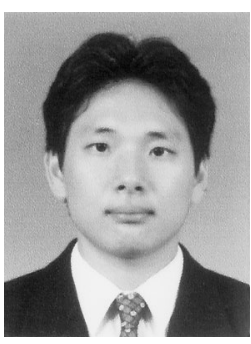

Sungwoo Cha was born in Seoul, Korea in 1976. He received the B.S. and M.S degrees from Kookmin University, Seoul, Korea, in 1998 and 2001, respectively. He is now working towards his Ph.D. degree at Osaka University. His current research interests include CMOS RF and analog circuits. Mr. Cha is a student member of the IEEE.

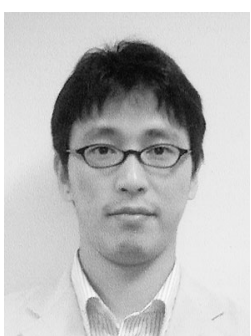

Tetsuya Hirose was born in Tottori, Japan in 1976. He received the M.S. and B.S. degrees from Osaka University, Osaka, Japan in 2000 and 2002, respectively. He is now working towards his Ph.D. degree at Osaka University. His current research interest is Analog Circuits.

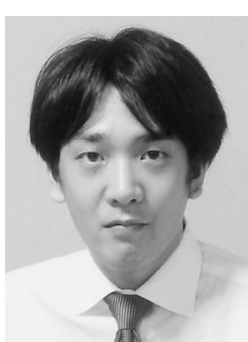

Masaki Haruoka was born in Hyogo, Japan in 1973. He received the B.S. and M.S. degrees from Himeji Institute of Technology, Hyogo, Japan, 1995 and 1997, respectively. He joined Technology Development and Researching Laboratry, Furuno Electric CO., LTD., Hyogo, Japan, where he contributed to the design of GPS RF front-end circuits from 1997. He is now working towards his Ph.D. degree at Osaka University. His current research interest includes CMOS RF circuits. Mr. Haruoka is a student

member of the IEEE. 


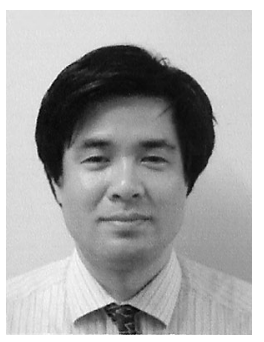

Toshimasa Matsuoka received the B.S., M.S. and Ph.D. degrees in electronic engineering from Osaka University, Osaka, Japan, in 1989, 1991 and 1996, respectively. During 1988-1991, he was involved in the research of heterostructures and superlattices of GaAs and related compounds. During 1991-1998, he worked for the Central Research Laboratories, Sharp Corporation, Nara, Japan, where he was engaged in the research and development of deep submicron CMOS devices and ultra thin gate oxides. Since 1999, he has been working for Osaka University, where he is Associate Professor now. His current research includes phase locked loops and CMOS RF circuits. Dr. Matsuoka is a member of the Japan Society of Applied Physics and the IEEE.

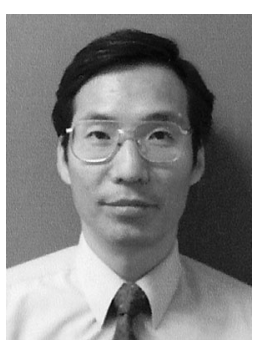

Kenji Taniguchi received the B.S., M.S. and Ph.D. degrees from Osaka University, Osaka, Japan, in 1971, 1973 and 1986, respectively. From 1973 to 1986, he worked for Toshiba Research and Development Center, Kawasaki, Japan where he was engaged in process modeling and the design of MOS LSI fabrication technology. He was a Visiting Scientist at Massachusetts Institute of Technology, Cambridge, from July 1982 to November 1983. Presently, he is a Professor of Electronics and Information Systems at Osaka University. His current research interests are in analog circuits, radio frequency circuits, device physics and process technology. Prof. Taniguchi is a member of the Japan Society of Applied Physics. He is a fellow of the IEEE. 